\title{
Laboratory-Scale Growth of Organic Crystals from the Melt
}

\author{
Steven P. Kelley \\ Department of Chemistry, University of Missouri, 601 S. College Ave., Columbia, MO 65211 \\ (kelleysp@missouri.edu)
}

Growth of suitable single crystals for structure determination remains a challenge in synthetic chemistry. Nearly all crystals used in structure determination are grown from solutions by methods which must be optimized empirically and which can take weeks to evaluate. By contrast, crystal growth from the melt is an extremely widely used and well developed technique in which high-quality single crystals are routinely grown at rates of grams per hour. Melt-based techniques are mainly used with inorganic systems but should be generally applicable to most compounds; however the equipment and techniques are designed for temperatures and masses which are infeasible for most laboratory-scale syntheses. An important exception is the widely used technique of hot-stage microscopy, but here the ability to isolate the sample is sacrificed for better environment and temperature control.

In this study, the Bridgman-Stockbarger technique is adapted to grow crystals under direct observation with an optical polarizing microscope. Crystals are heated by simple contact of their container with a resistive wire and moved by hand. Observation of crystal growth and melting in real time using the microscope allows the operator to compensate for the lack of temperature gradient control. This approach combines two widely used techniques, melt-recrystallization and hot-stage microscopy, in a way which can be used to grow, isolate, and manipulate single crystals.

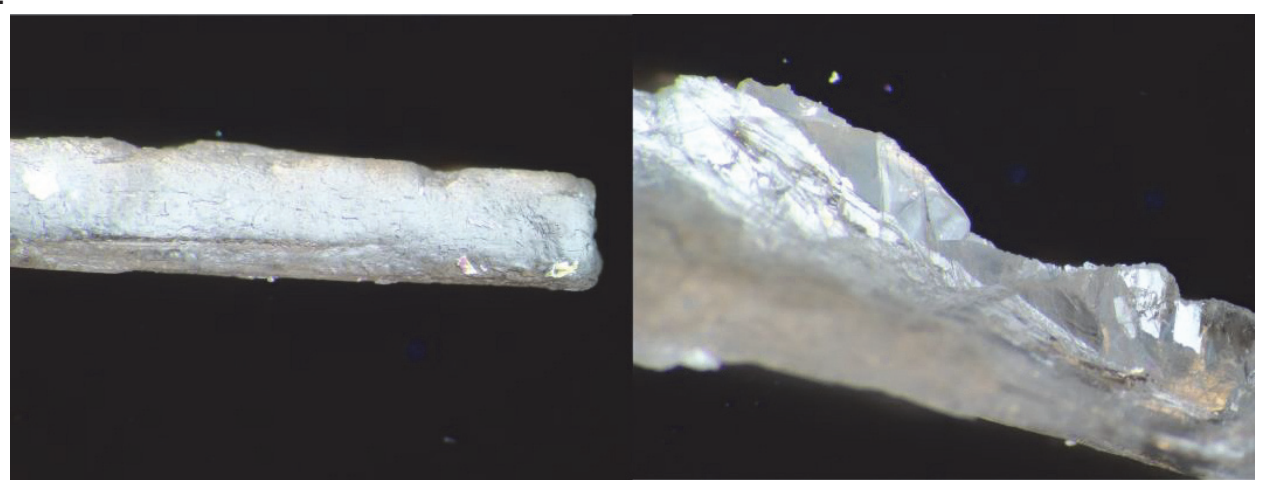

Fig. 1. Solution-grown (left) and melt-recrystallized (right) crystals of camphor monobromide illuminated under transmission with polarized light. 